\section{Asthma-Präventionsstudie ante portas}

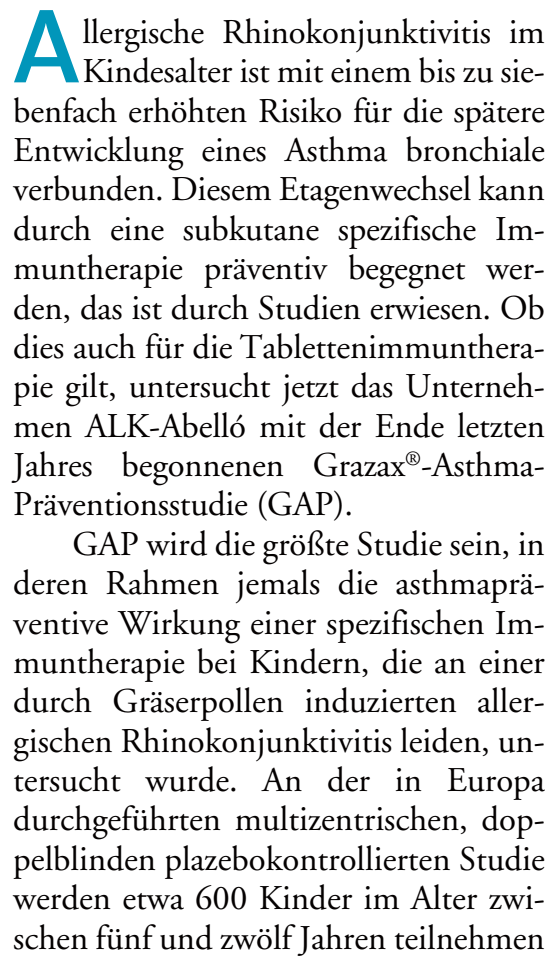

obachtet, um nachweisen zu können, ob die Wirkung nach Abschluss der Behandlung erhalten bleibt.

Erkka Valovirta, GAP-Forschungsleiter aus Turku, Finnland, betonte: „Zahlreiche an allergischer Rhinokonjunktivitis erkrankte Kinder entwickeln im weiteren Krankheitsverlauf Asthma, was eine erhebliche Gesundheitsbelastung darstellt und häufig zu krankheitsbedingten Fehltagen in der Schule, eingeschränkter schulischer Leistung, häufigen Arztbesuchen und sogar Krankenhausaufenthalten führt. Bei positivem Ausgang kann diese Studie die Art und Weise, wie an Heuschnupfen erkrankte Kinder in Zukunft behandelt werden, erheblich verändern. Zum ersten Mal werden wir anhand evidenzbasierter Studienergebnisse zeigen können, dass die immuntherapeutische Behandlung einer Gräserpollenallergie mit der Tablette Grazax $^{\circledR}$ das Fortschreiten der Erkrankung zu Asthma verhindert."

Nach Informationen von ALK-Abelló Hørsholm, Dänemark

\title{
Hochleistungstherapie auch für Kinder
}

$\mathrm{H}$ eute gehört die sublinguale Immuntherapie (SLIT) fest zum allergologischen Behandlungsalltag und empfiehlt sich insbesondere auch für Kinder. Eine wirksame und gut praktikable Therapieoption steht mit AllerSlit ${ }^{\circledR}$ forte zur Verfügung. Nachdem sich diese sichere und gut verträgliche Hochleistungs-SLIT bereits bei Erwachsenen mit allergischer Rhinokonjunktivitis in der klinischen Prüfung sowie in der Praxis bewährt hat, liegen nun auch aktuelle, noch nicht publizierte Ergebnisse zur Anwendung von AllerSlit ${ }^{\circledR}$ forte Gräser bei Kindern vor: 79 Studienteilnehmer im Durchschnittsalter von neun Jahren hatten über einen Zeitraum von 17 Monaten einmal täglich AllerSlit ${ }^{\circledR}$ forte angewandt und während des Gräserpollenflugs ihre Symptome und den Medikamentenverbrauch dokumentiert. Am Ende der Behandlung waren die Kinder nahezu symptomfrei und brauchten kaum noch Medikamente. Der Symptom-Medikations-Score näherte sich dem Wert Null. Die Kinder vertrugen die Hochleistungs-SLIT gut, Nebenwirkungen traten kaum auf.

AllerSlit ${ }^{\circledR}$ forte ist mit einem Allergengehalt von 21,6 $\mu \mathrm{g}$ laut CREATEEssay $(40 \mu \mathrm{g}$ in unternehmenseigenem Essay) für das Gräser-Gruppe-5-Allergen in der Erhaltungsdosis die am höchsten dosierte SLIT, die auf dem deutschen Markt erhältlich ist. Mit ihrer kurzen Aufdosierungszeit von nur einer halben Stunde lässt sich die Hochleistungs-SLIT auch gut in den Praxisablauf integrieren, wie im Rahmen einer Anwendungsbeobachtung bestätigt wurde. Dabei wurden mit Hilfe eines strukturierten Fragebogens die Daten von 345 Patienten mit Gräserpollenallergie zwischen fünf und 75 Jahren durch 83 niedergelassene Allergologen erfasst. Die Aufdosierung mit AllerSlit ${ }^{\circledR}$ forte erfolgte bei der Hälfte der Patienten durch ein zweimaliges Verdoppeln der Dosis (ein, zwei und vier Tropfen) mit Wartezeiten von jeweils $15 \mathrm{Mi}$ - nuten. Es traten nur leichte bis moderate Nebenwirkungen auf, insbesondere das orale Allergiesyndrom. 95\% der Allergologen gaben an, die Aufdosierung problemlos oder nur mit geringem zusätzlichen Aufwand in den Praxisalltag integrieren zu können. Auch bei den Patienten kam die rasche Aufdosierung gut an.

Auch eine hochdosierte SLIT wirkt nur, wenn die Compliance des Patienten stimmt. Bei Kindern sollte der Arzt daher bei der Aufdosierung die gute Lernfähigkeit der kleinen Patienten nutzen und ein Ritual einüben. „Bei der sublingualen Immuntherapie mit Kindern hat sich in der Praxis gezeigt, dass sie selten Probleme mit der Compliance aufweisen. Wenn sie die Notwendigkeit der täglichen Anwendung verstanden haben, nehmen sie die Einnahme sehr ernst und es ist mit einem guten Therapieerfolg zu rechnen", berichtete Prof. Dr. Ulrich Wahn, Berlin.

Nach Informationen von Allergopharma, Reinbek 